\title{
Supramolecular Catalysis of $m$-Xylene Isomerization by Cucurbiturils: Transition State Stabilization, Vibrational Coupling, and Dynamic Binding Equilibrium
}

\author{
Hugues Lambert, Yong-Wei Zhang,* and Tung-Chun Lee*
}

Cite This: https://dx.doi.org/10.1021/acs.jpcc.0c02012

Read Online

ABSTRACT: The ability of cucurbit[6] uril (CB6) and cucurbit[7]uril (CB7) to catalyze the thermally activated 1,2-methyl shift isomerization pathway of $m$-xylene in vacuum is investigated using infrequent metadynamics. CB6 is predicted to effectively and selectively catalyze the meta-to-para isomerization through stabilization of the transition state (TS) by van der Waals push (packing coefficient $\approx 74 \%$ ), while inhibiting the meta-to-ortho pathway by molding effects of the cavity. Interestingly, despite the snug binding, a very low rate of host-guest vibrational energy transfer is revealed using a novel approach of hostguest partition of the mode-specific anharmonic relaxation rates and $a b$

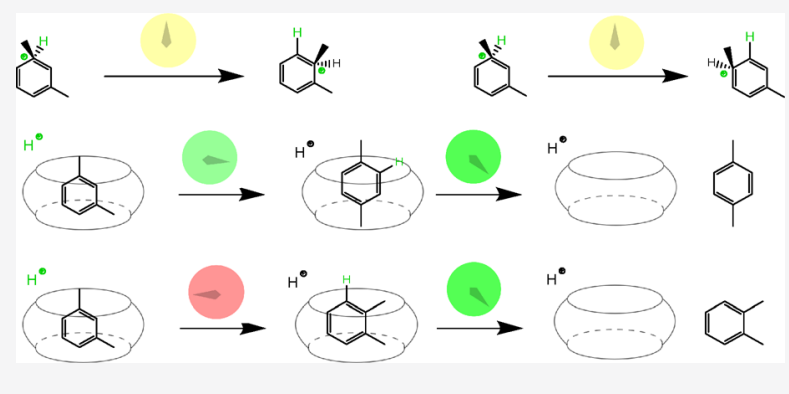
initio molecular dynamics. The weak vibrational coupling suggests that $\mathrm{CB}$ can act as a thermal buffer, possibly shielding encapsulated guests from outside vibrational perturbations such as solvent effects. This dynamic effect could provide an additional boost to the reaction rate by blocking the occurrence of reaction barrier recrossing caused by the friction with surrounding molecules. Finally, mean residence times of xylene into the hosts' cavity were estimated for a range of host-guest complexes, revealing a highly dynamic equilibrium allowing very high guest turnover rates that could minimize catalyst inhibition effects commonly suffered by other supramolecular catalysts.

\section{INTRODUCTION}

Modulation of reaction rates and selectivity has always been a core objective in chemistry. During the last 2 decades, it has become increasingly clear that confining the reaction volume in one or more dimensions to sizes of the same order of magnitude as the reacting molecule has the potential to favor or inhibit specific reaction pathways. The physical effects driving this reactivity shift include local enrichment in reactants through preferential interaction with the interior of the confined environment ${ }^{1}$ or faster diffusion kinetics. ${ }^{2}$ Preorganization of a reagent inside the confined space can also end up favoring a specific reaction pathway. ${ }^{3}$ Stabilization of the transition state, through electrostatic and van der Waals interactions with the cavity, is able to shift, sometimes dramatically, ${ }^{4}$ the height of reaction barriers. This effect is expected to contribute significantly to the catalytic efficiency of enzymes. ${ }^{5}$ Charge transfer from the confining environment toward the confined reagent, resulting in variation in bond polarization, is also known to allow for large shifts in energy barriers. ${ }^{6}$ An in-depth review of these effects in the case of carbon nanotubes can be found elsewhere. ${ }^{7}$ While confinement in one and two dimensions has been mostly limited to, respectively, sandwiches of $2 \mathrm{D}$ materials and carbon nanotubes, confinement in three dimensions yielding "0D" systems has been performed in a much larger range of systems including nanoflasks, ${ }^{3}$ protein complexes, ${ }^{8}$ nanocages, ${ }^{9}$ polymer assemblies, ${ }^{10}$ and macrocycles. ${ }^{11}$

Cucurbit $[n]$ urils $(\mathrm{CB} n$ where $n=5-8)$ are a class of artificial organic macrocycles that are made of repeating units of glycoluril. Besides being famous for forming host-guest complexes with exceptionally strong binding affinities ${ }^{12,13}$ rivalling that of the avidin-biotin pair, cucurbiturils have been shown to possess useful catalytic properties in a range of systems. Indeed, spectacular rate enhancement ${ }^{14,15}$ and inhibition of unwanted reactions ${ }^{16,17}$ in addition to substrate preorganization ${ }^{18}$ have been reported among others. Cucurbiturils' peculiar properties, including a well-defined shape and hydrophobic cavity along with their chemical stability and UV transparency ${ }^{19}$ have powered numerous applications in catalysis in recent years. ${ }^{20}$

Experimental reports of catalysis in confined environments are often supplemented with ab initio studies to attempt a quantification of the relative strength of interactions between the substrate and its surroundings, the relative stability of

Received: March 6, 2020

Revised: April 5, 2020

Published: April 6, 2020 


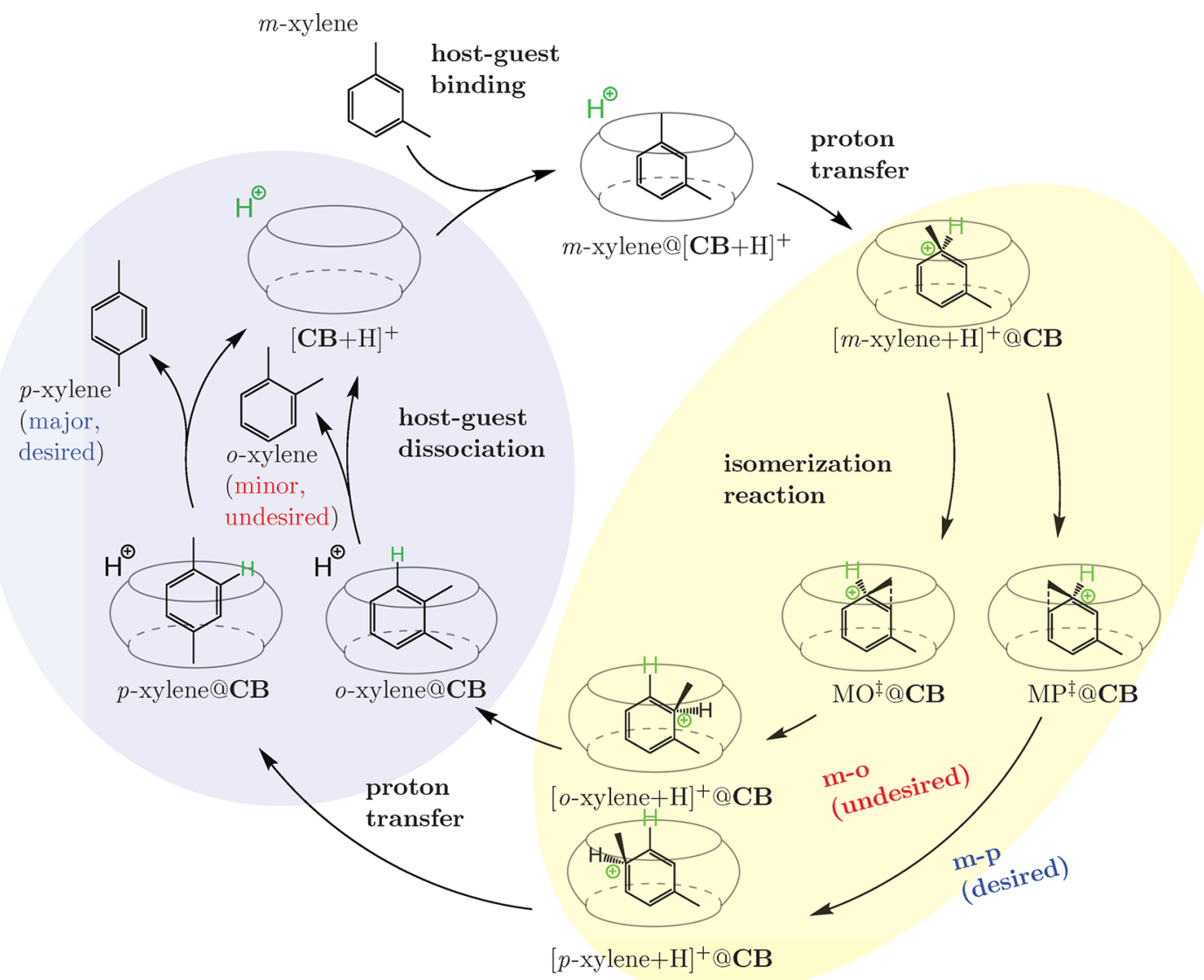

Figure 1. Overview of the role of $\mathbf{C B}$ in the catalysis of $m$-xylene. After a complex forms between a protonated CB and $m$-xylene, a proton is transferred from the portal region of $\mathbf{C B}$ toward a methyl bearing carbon in $m$-xylene, yielding $[m \text {-xylene }+\mathrm{H}]^{+}$. This protonated xylene is then able to isomerize to either $[p \text {-xylene }+\mathrm{H}]^{+}$or $[o \text {-xylene }+\mathrm{H}]^{+}$, forming, respectively, the complexes $[p \text {-xylene }+\mathrm{H}]^{+} @ \mathbf{C B}$ and $[o \text {-xylene }+\mathrm{H}]^{+} @ \mathbf{C B}$ via the transition state denoted as $\mathrm{MP}^{\ddagger} @ \mathbf{C B}$ and $\mathrm{MO}^{\ddagger} @ \mathbf{C B}$, respectively. The protonated xylene transfers back a proton to the portal region of $\mathbf{C B}$ and subsequently exits the CB cavity. The free CB host is again free to accommodate $m$-xylene and repeat the cycle. The isomerization reaction is shaded in yellow on the right side of the scheme while the host-guest dissociation process is shaded in blue on the left-hand side.

different system configurations, ${ }^{18}$ and the energies of the possible transitions states. ${ }^{21}$ Usually, these computations rely on direct optimization of the system in order to locate minima on the potential energy surface (PES) or first order saddle points corresponding to transition states. This approach has been very successful in predicting energy barriers in simple systems where the PES is smooth. ${ }^{22}$ It is however known that in systems with multiple degrees of freedom, the PES can become very rough, resulting in multiple local minima and saddle points. ${ }^{23}$ In addition, as direct minimization converges to a single point in the atomic configuration space, much of the information about the surrounding energy landscape and its curvature is inaccessible, preventing the study of most entropic effects. $^{24}$

A straightforward solution to the problems, essentially due to sampling, encountered during direct optimization of the system, would be to use $a b$ initio molecular dynamics (AIMD). By letting reactions occur spontaneously, thus enabling direct comparison of reaction rates, the reliable sampling of the most statistically favored states is enforced. The main obstacle to this approach is that the required simulation times for reactions with a large energy barrier would be unacceptably long, rendering AIMD prohibitively expensive. ${ }^{25} \mathrm{~A}$ number of techniques have been proposed to encourage the system to explore the higher energy regions associated with chemical reactions of interest, including umbrella sampling, ${ }^{26}$ chemical flooding, ${ }^{27}$ adaptive force bias, ${ }^{28}$ and metadynamics. ${ }^{25}$ In particular, metadynamics has been used to resolve the multiple pathways through which a system is able to react ${ }^{29}$ and characterize the intricate binding and unbinding dynamics of small molecules with macromolecular receptors. ${ }^{30,31}$ The versatility and generality of metadynamics appears to be well suited to the challenging task of characterizing the impact of a confined environment on the reactivity of small molecules.

In this work, we investigate thermally activated isomerization reactions of $m$-xylene inside the $\mathrm{CB}$ cavity using an infrequent metadynamics approach. This approach offers a precise and accurate estimation of the free energy barrier height despite the rough and shallow potential energy surface of the host-guest system, which cannot be achieved via conventional direct optimization of the transition states. Our results indicate that CB6 can effectively and selectively catalyze the meta-to-para isomerization of xylene through stabilization of the transition state (TS) by van der Waals push (packing coefficient $\approx 74 \%$ ), while inhibiting the meta-to-ortho pathway by molding effects of the cavity. Catalytic effects become less pronounced in the case of CB7 which has a larger cavity volume, resulting in a small packing coefficient of $\approx 43 \%$. Interestingly, host-guest vibrational energy transfer was computed to be exceptionally slow in the case of CB6 despite the snug binding, based on host-guest partition of the modespecific anharmonic relaxation rates and $a b$ initio molecular 
dynamics. This dynamic effect could provide additional boost to the reaction rate by blocking the occurrence of reaction barrier recrossing caused by the friction with surrounding molecules in the media. Finally, the escape time of the three xylene isomers from the $\mathrm{CB}$ cavity was estimated using infrequent metadynamics, revealing a small residence time, smaller than that of the average reaction time at high temperature, thus hinting at $\mathrm{CB} 6$ and $\mathrm{CB} 7$ probable resilience to poisoning and deactivation as a catalyst.

\section{METHOD}

The present investigation focuses on the isomerization and dissociation step as illustrated in Figure 1. Computational details are described in the Supporting Information.

Initially, some of the challenges associated with estimating the energy barriers to reaction using direct optimization of transition states are illustrated using the $[m \text {-xylene }+\mathrm{H}]^{+}$to $[o$ xylene $+\mathrm{H}]^{+}\left(\mathrm{MO}^{\ddagger}\right)$ transition state in the $\mathrm{CB} 7$ system $\left(\mathrm{MO}^{\ddagger} @ \mathrm{CB} 7\right)$. From an optimized configuration, a range of slightly perturbed initial geometries are constructed by rotating and then shifting the $[m \text {-xylene }+\mathrm{H}]^{+}$guest inside the cavity by a series of vectors $(a, b, c)$ in the $x, y, z$ direction as illustrated in Figure S1a.

Following the observation that several transition states are obtained, infrequent metadynamics is used to estimate the thermodynamic quantities defining the $[m \text {-xylene }+\mathrm{H}]^{+}$to $[p$ xylene $+\mathrm{H}]^{+}$and $[o \text {-xylene }+\mathrm{H}]^{+}$isomerization reactions, namely, the enthalpy $\left(\Delta H^{\ddagger}\right)$ and entropy $\left(\Delta S^{\ddagger}\right)$ of reaction. Conceptually, starting from the systems $[m \text {-xylene }+\mathrm{H}]^{+} @$ CB6 and $[m \text {-xylene }+\mathrm{H}]^{+} @$ CB7 in equilibrium at several temperatures, a potential bias $V_{\text {bias }}$ is gradually applied on the collective variables $C_{1}$ and $C_{2}$ as defined in Figure $S 1 \mathrm{~b}$. After a given time, written $t^{\mathrm{MTD}}$, the forces associated with the accumulated potential coerce the system into changing configuration, in this case changing from the meta to the ortho or para isomer depending on the definition of $C_{2}$ (i.e., eq $\mathrm{S} 2$ for the $\mathrm{MO}^{\ddagger} \mathrm{TS}$ and eq S3 for the $\left.\mathrm{MP}^{\ddagger} \mathrm{TS}\right)$. Now instead of estimating the free energy of the system through direct use of the free energy surface (FES) as in standard metadynamics, ${ }^{29}$ an indirect approach based on the extraction of the free energy from the observed reaction rates is chosen. ${ }^{32}$ Indeed, it has been suggested that the free energy barriers obtained from partially converged free energy surfaces were sensitive to the choice of the rate of application of the potential bias. ${ }^{33}$ In the procedure selected, the metadynamic time $t^{\mathrm{MTD}}$ is translated to an unbiased equivalent $t^{0}$ through the use of an acceleration factor $\alpha$ according to ${ }^{34}$

$$
\begin{aligned}
& t^{0}=\alpha t^{\mathrm{MTD}} \\
& \alpha=\sum_{i=0}^{N} \Delta t_{i}^{\mathrm{MTD}} \mathrm{e}^{V_{\text {bias }}\left(q, t_{i}\right)}
\end{aligned}
$$

where $\Delta t_{i}^{\mathrm{MTD}}$ stands for the biased trajectory time step, usually constant and of the order of 1 fs. Average reaction times toward $\mathrm{MO}^{\ddagger}$ and $\mathrm{MP}^{\ddagger}$ are then compiled for 120 trajectories for each system (in vacuum, in CB6 and in CB7) at several temperatures and thermodynamic quantities are extracted by fitting $^{32}$ the average reaction times and temperatures to the Eyring equation. ${ }^{35}$

$$
\ln \frac{k}{T}=\frac{-\Delta H^{\ddagger}}{R} \frac{1}{T}+\ln \frac{\kappa k_{\mathrm{B}}}{h}+\frac{\Delta S^{\ddagger}}{R}
$$

where $T$ is the temperature in Kelvin and $k$ is the apparent reaction rate in $\mathrm{s}^{-1}$. The quantity $\kappa$ is known as the transmission coefficient and $\Delta S^{\ddagger}$ and $\Delta H^{\ddagger}$ represent, respectively, the activation entropy and the activation enthalpy. The ideal gas constant, Planck's constant, and the Boltzmann's constant are, respectively, written as $R, h$, and $k_{\mathrm{B}}$. The infrequent metadynamics investigation demonstrates the promising role of CB6 as a catalyst for the isomerization of $[m \text {-xylene }+\mathrm{H}]^{+}$and clearly reveals the limitations of using direct optimizations to estimate reaction barrier heights.

To estimate the effectiveness of heat transfer between the host and the guest, a vibrational analysis is then performed. The vibrational coupling between $\mathbf{C B 6}$ and $[m \text {-xylene }+\mathrm{H}]^{+}$is estimated by first computing the anharmonic decay rates of the vibrational modes of the complex. Then, by identifying modes predominantly located on $[m \text {-xylene }+\mathrm{H}]^{+}$and $\mathrm{CB} 6$ as well as those spread over both, the contribution of the xylene located modes to the overall decay rates is computed.

To validate the approach, a mode predominantly located on CB6 and predicted to possess a negligible decay rate toward xylene-located modes is further investigated using molecular dynamics. The eigenvector corresponding to a mode overwhelmingly located on the CB6 host is selected and $1 \mathrm{kcal} / \mathrm{mol}$ is deposited on that mode as kinetic energy from the equilibrium position. The system is then allowed to propagate in time for $1 \mathrm{~ns}$, and the amplitude of the various vibrational modes is sampled over the trajectory in order to follow the energy redistribution process.

Finally, the residence time of the $m, o$, and $p$-xylene isomers is estimated via infrequent metadynamics. The single collective variable used in a given host-guest system corresponds to the distance between the centroids of the atomic positions of the cucurbituril host and those of the guest. The unbiased unbinding time is similarly computed using the acceleration factor as defined in eq 2 .

\section{RESULTS AND DISCUSSION}

Isomerization of $[m \text {-xylene }+\mathrm{H}]^{+}$. Finding a transition state in a complex energy surface such as the inside of the relatively large CB7 cavity can be a challenge. Indeed, the 1188 starting geometries used to perform a direct TS optimization in the cavity converged toward markedly different TS in terms of energy. As shown in Figure S2, the empirical cumulative distribution function of the TS energies indicates that they are relatively uniformly distributed between 22.5 and $32 \mathrm{kcal} / \mathrm{mol}$. This near $10 \mathrm{kcal} / \mathrm{mol}$ spread illustrates the difficulty of quantifying the interaction energy of the transition state with the cavity. Performing a direct TS optimization on a single $\mathrm{MO}^{\ddagger} @ \mathrm{CB} 7$ configuration could in principle lead to an unacceptable sampling error as large as $10 \mathrm{kcal} / \mathrm{mol}$ and possibly higher. One way to go around this problem would be to sample a large number of transition states inside the cavity and compute their relative probability of occurrence using a Boltzmann distribution. ${ }^{36}$ Using this probability distribution, one could compute the Boltzmann averaged energy and take into account the information contained in the cavity's energy landscape. It can be expected that the sampling problem can only be partially solved this way as it stays heavily reliant on the starting TS guesses provided by the practitioner. For example, it is known that CB7 can change shape to accommodate a guest further complicating the selection and generation of the starting configurations. Such an approach would also not be very general as intimate knowledge of the 
(a)

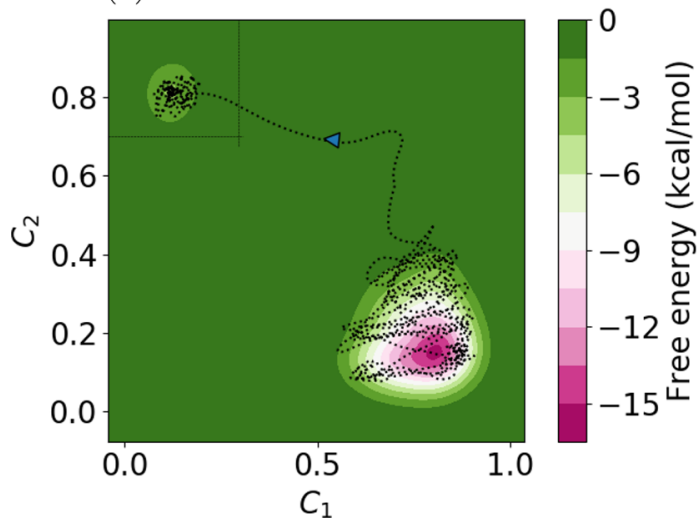

(b)
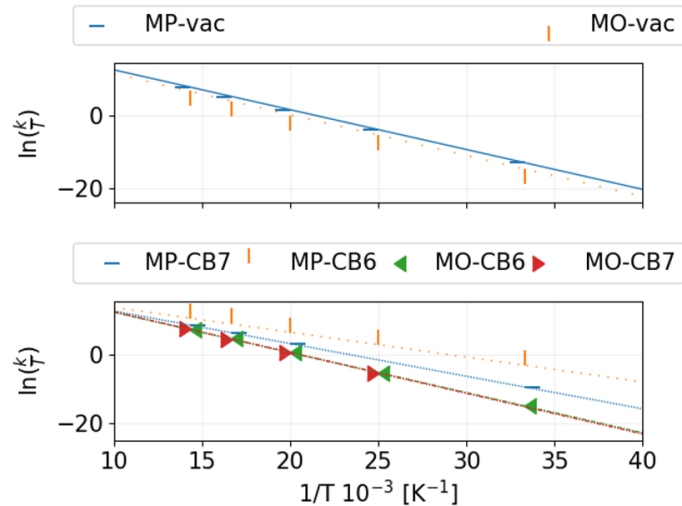

Figure 2. (a) Free energy surface obtained using a biased trajectory in the case of the MP $@$ @B6 system. The free energy surface corresponds to the accumulated potential until the system escapes the initial potential well located around $(0.8,0.2)$ and enters a new potential well located in $(0.2$, $0.8)$. The time to reaction $t^{\mathrm{MTD}}$ is defined as the time needed for the system's collective variables to enter the square marked on the upper left of the figure in $[0,0.7] \times[0.3,1]$. It can be seen that as both the height of the Gaussians and their rate of deposition are relatively low, the trajectory of the reagent in the initial free energy basin located near $C_{1}=0.8$ and $C_{2}=0.2$ is diffusive. The free energy surface shown is constructed using the accumulated potential of the first 45 ps while the dashed trajectory starts at 40 ps and lasts 2 ps. The collective variables $C_{1}$ and $C_{2}$ are defined in eq S2 and eq S3. (b) Eyring plots obtained by fitting the simulated data points to eq 3. Each data point represents the average time obtained by fitting the cumulative distribution of reaction times at the associated temperature with a cumulative Poisson distribution as shown in Figure S3. Averaged rates corresponding to times with a $p$-value superior to $5 \times 10^{-2}$ were included (see Table 2). Note that the data points for $\mathrm{MO}^{\ddagger}$-vac (respectively, $\mathrm{MP}^{\ddagger}$-CB6) are represented by the top (respectively, bottom) of the orange vertical line marker. It can be seen that the fitted curves almost overlap in the case of the $\mathrm{MO}^{\ddagger}$ and $\mathrm{MP}^{\ddagger}$ isomerization. Similarly, the $\mathrm{MO}^{\ddagger}$ isomerization pathway occurs at a similar rate whether in $\mathrm{CB} 6$ or $\mathrm{CB} 7$. On the other hand, the $\mathrm{MP}^{\ddagger}$ isomerization in $\mathrm{CB} 6$ shows by far the highest isomerization rate at all temperatures while the $\mathrm{MP}^{\ddagger}$ isomerization in $\mathrm{CB} 7$ occurs at an intermediate rate.

system and its binding modes with guests would be needed to generate a high quality set of starting configurations.

A common answer to the sampling problem is found in molecular dynamics. In the limit of very long times, the system should sample all configurations including the high energy ones. As prohibitively long simulation times would be needed for unbiased molecular dynamics simulations to overcome barriers over a few $\mathrm{kcal} / \mathrm{mol}$, biasing techniques are often used to allow high energy phenomena, such as chemical reactions, to be studied. ${ }^{25}$

The times to reaction $t^{\mathrm{MTD}}$ were collected and converted to unbiased times through the use of eq 2. A sample free energy surface obtained from a trajectory corresponding the $\mathrm{MO}^{\ddagger} @$ CB6 system is shown in Figure 2a along with the reactive part of the trajectory in overlay. The time to reaction $t^{\mathrm{MTD}}$ is defined as the time needed for $C_{1}$ to become lower than 0.7 and for $C_{2}$ to become higher than 0.3. If $C_{1}$ becomes lower than 0.3 while $C_{2}$ is still lower than 0.3 , the trajectory is discarded as it is assumed that a competing side reaction, such as a hydrogen transfer instead of a methyl transfer, occurred. As an illustration, Videos 1, 2, 3, and 4 showing the isomerization of $\mathrm{MO}^{\ddagger}$ and $\mathrm{MP}^{\ddagger}$ in vacuum and in CB6 are shown in the Supporting Information.

An average time is obtained for each subsystem by fitting the corresponding reaction rate $k$ to the temperature via an Eyring plot as shown in Figure $2 \mathrm{~b}$ using eq 3 . The average times are computed by fitting the cumulative distribution function of an exponential to the empirical time distribution ${ }^{37}$ as illustrated in Figure S3. The activation enthalpies $\Delta H^{\ddagger}$ and entropies $\Delta S^{\ddagger}$ (including translational, rotational, and vibrational contributions) are shown in Table 1 while fitted times to reaction are shown in Table S1. It should be noted that the experimental decomposition temperature of CB6 is $697 \mathrm{~K}$ and CB7 is 643 $\mathrm{K}$, rendering values obtained at $700 \mathrm{~K}$ physically inaccessible. This degradation is expected to take place on a timescale much
Table 1. Thermodynamical Data Extracted from the Plot Shown in Figure $2 b$ Using eq 3 are Shown as $\Delta H_{\mathrm{MTD}}^{\ddagger}$ and $\Delta S_{\text {MTD }}^{\ddagger}$ while Values Obtained from Direct Optimization of the Transition State in Vacuum are Shown as $\Delta H_{\mathrm{Opt}}^{\ddagger}$ and $\Delta S_{\mathrm{Opt}}^{\ddagger}$

\begin{tabular}{lcccc}
\multicolumn{1}{c}{ system } & $\begin{array}{c}\Delta H_{\mathrm{MTD}}^{\ddagger} \\
(\mathrm{kcal} / \mathrm{mol})\end{array}$ & $\begin{array}{c}\Delta S_{\mathrm{MTD}}^{\ddagger} \\
(\mathrm{cal} / \mathrm{mol} / \mathrm{K})\end{array}$ & $\begin{array}{c}\Delta H_{\mathrm{Opt}}^{\ddagger} \\
(\mathrm{kcal} / \mathrm{mol})\end{array}$ & $\begin{array}{c}\Delta S_{\mathrm{Opt}}^{\ddagger} \\
(\mathrm{cal} / \mathrm{mol} / \mathrm{K})\end{array}$ \\
$\mathrm{MP}^{\ddagger}$-vac & 21.56 & -1.23 & 22.88 & -1.12 \\
$\mathrm{MP}^{\ddagger} @ \mathrm{CB} 6$ & 14.25 & -6.11 & 14.08 & -69.18 \\
$\mathrm{MP}^{\ddagger} @ \mathrm{CB} 7$ & 18.72 & -3.70 & 20.24 & -50.72 \\
$\mathrm{MO}^{\ddagger}$-vac & 22.14 & -2.74 & 24.22 & -1.32 \\
$\mathrm{MO}^{\ddagger} @ \mathrm{CB6}$ & 23.07 & -0.07 & 29.12 & -65.77 \\
$\mathrm{MO}^{\ddagger} @ \mathrm{CB7}$ & 23.38 & 0.47 & 26.32 & -51.69 \\
\hline
\end{tabular}

larger than the current simulations and is not a concern for the current investigation.

The barrier heights for the isomerization of $[m \text {-xylene }+\mathrm{H}]^{+}$ to $[o \text {-xylene }+\mathrm{H}]^{+}$in $\mathbf{C B} 7$ obtained from the Eyring plot and shown in Table 1 of $23.38 \mathrm{kcal} / \mathrm{mol}$ compares favorably with the lower end of the energy window obtained from direct optimization of $\mathrm{MO}^{\ddagger} @ \mathrm{CB} 7$ as shown in Figure S2 with the lowest $\mathrm{MO}^{\ddagger} @ \mathrm{CB} 7$ energy reaching $22.67 \mathrm{kcal} / \mathrm{mol}$. Particularly significant is the large decrease in $\Delta H_{\mathrm{MTD}}^{ \pm}$from 21.56 $\mathrm{kcal} / \mathrm{mol}$ in the case of $\mathrm{MP}^{\ddagger}$-vac to about $14.25 \mathrm{kcal} / \mathrm{mol}$ in the case of MP $¥$ @CB6. This effect is assumed to originate from a stabilization of the transition state through favorable "pushing" van der Waals forces. ${ }^{11}$ Indeed the molecular volume of $m$-xylene is $125 \AA^{3}$ which indicates a relatively high packing coefficient in the $164 \AA^{3}$ cavity of CB6. ${ }^{38}$ This large guest size relative to the cavity is suspected to induce a compression effect akin to a pressure increase. ${ }^{39}$ A high pressure is susceptible to increase the rate of reactions for which the volume of activation is negative, which is consistent with a $m$ xylene volume decrease to $122 \AA^{3}$ (packing coefficient of 
$\approx 74 \%$ ) in the case of $\mathrm{MP}^{\ddagger}$. The "pushing" van der Waals forces are not observed in the case of CB7 due to its significantly larger cavity of $282 \AA^{3}$, corresponding to a packing coefficient for $\mathrm{MP}^{\ddagger}$ of $\approx 43 \%$. Besides the absolute volume of the transition state, its shape has been shown to play a significant role as well which is why $\mathrm{MO}^{\ddagger}\left(121 \AA^{3}\right)$, with a packing coefficient of $\approx 75 \%$ in CB6, is not stabilized despite possessing a volume similar to $\mathrm{MP}^{\ddagger}$.

While a reasonable correlation for $\Delta H_{\mathrm{MTD}}^{\ddagger}$ with $\Delta H_{\mathrm{Opt}}^{\ddagger}$ is obtained both for the isomerization in vacuum and inside of the cucurbiturils, there is a marked discrepancy between the values obtained for $\Delta S_{\mathrm{MTD}}^{\ddagger}$ and $\Delta S_{\mathrm{Opt}}^{\ddagger}$. Unlike the estimate for $\Delta H_{\mathrm{MTD}}^{\ddagger}$, it is expected that $\Delta S_{\mathrm{MTD}}^{\ddagger}$ will be dependent on the update frequency of the metadynamic bias, with longer strides translating into longer apparent times to dissociation. Long times to reaction would translate to smaller reaction rates and in fine result in a larger apparent entropy barrier to reaction. Also in the case of $\mathrm{MO}^{\ddagger}$-vac and $\mathrm{MP}^{\ddagger}$-vac where the potential energy surface is expected to be smoother and therefore a direct TS optimization can be performed, it appears that the barrier height and entropy of activation computed by metadynamics $\Delta H_{\text {MTD }}^{\ddagger}$ and $\Delta S_{\text {MTD }}^{\ddagger}$ are very close to the values obtained by direct optimization $\Delta H_{\mathrm{Opt}}^{\ddagger}$ and $\Delta S_{\mathrm{Opt}}^{\ddagger}$, with discrepancies lower than $1 \mathrm{kcal} / \mathrm{mol}$ in the case of $\Delta H^{\ddagger}$ and close to $1 \mathrm{cal} / \mathrm{mol} / \mathrm{K}$ in the case of $\Delta S^{\ddagger}$.

Even accounting for the fact that the PM6-D3 level of theory is not intended for chemical accuracy, those values compare favorably with the experimental values for both $\mathrm{MP}^{\ddagger}$-vac $\left(\Delta H^{\ddagger}\right.$ $\left.=20.9 \mathrm{kcal} / \mathrm{mol} ; \Delta S^{\ddagger}=-2.41 \mathrm{cal} / \mathrm{mol} / \mathrm{K}\right)$ and $\mathrm{MO}^{\ddagger}$-vac $\left(\Delta H^{\ddagger}=22.3 \mathrm{kcal} / \mathrm{mol} ; \Delta S^{\ddagger}=-0.68 \mathrm{cal} / \mathrm{mol} / \mathrm{K}\right)$ in the case of catalysis by hydrogen bromide. ${ }^{40}$ Protonated xylenes were also computed to have similar TS energies in acidic mordenite. ${ }^{21}$ Absolute reaction rates at $300 \mathrm{~K}$ for the case of $\mathrm{MO}^{\ddagger}(1.23 \times$ $\left.10^{-4} \mathrm{~s}^{-1}\right)$ and $\mathrm{MP}^{\ddagger}\left(6.94 \times 10^{-4} \mathrm{~s}^{-1}\right)$ isomerization in vacuum compare well with those adapted from the case of catalysis ${ }^{40}$ by $\mathrm{HBr}$ and $\mathrm{Al}_{2} \mathrm{Br}_{6}$ of $2.34 \times 10^{-4} \mathrm{~s}^{-1}$ for $\mathrm{MO}^{+}$and $1.05 \times 10^{-3}$ $\mathrm{s}^{-1}$ for $\mathrm{MP}^{\ddagger}$ (the second order reaction rates provided by the reference were adapted to a pseudo-first order reaction rate by using a fixed concentration of $1.2 \mathrm{~mol} / \mathrm{L}$ of $\mathrm{Al}_{2} \mathrm{Br}_{6}$ as in the experimental setting of the paper). On the other hand, reaction rates at $523-673 \mathrm{~K}$ in the case of a range of zeolites ${ }^{41,42}$ were of the order of $10 \mathrm{~h}^{-1}$ per site, which is order of magnitudes lower than the present estimates in CB6 and CB7 (over $3.6 \times$ $10^{8} \mathrm{~h}^{-1}$ at $600 \mathrm{~K}$ in all cases).

This massive discrepancy in site-specific turnover rate between the experimental zeolite values and the present $\mathrm{CB}$ estimates indicates that, for one, the reverse isomerization reaction needs to be accounted for to obtain the net reaction rate at high temperature. This is unlike at low temperature where the pathway with the lowest activation energy can be expected to dominate the direction of evolution of the system. Second, it suggests that most catalytic sites are probably inactive or inaccessible as accounting for the rates of the reverse reaction is not expected to dramatically alter the order of magnitude of the overall rate. ${ }^{42}$ Diffusion limitations and accessibility of acidic sites are also known to occur in zeolites and most likely constrain their maximum catalytic potential. ${ }^{43}$ Ultimately the effective catalytic rate achievable with CB6 depends on the form under which the macrocycle is used which will determine the fraction of cavities that is available to accommodate guests. It may well be that at high temperatures and pressures, the diffusion coefficient of the xylenes isomers is high enough to allow direct use of crystalline cucurbiturils.
Selectivity at temperature $T$, written $\mathrm{p} / \mathrm{o}(T)$, for $p$-xylene computed as in eq 4 , is expected to vary from 1 (100\% of product is $p$-xylene) at $300 \mathrm{~K}$ to 0.98 at $600 \mathrm{~K}$ in the case of CB6 and from 0.996 at $300 \mathrm{~K}$ to 0.85 at $600 \mathrm{~K}$ for CB7.

$$
\mathrm{p} / \mathrm{o}(T)=\frac{\frac{1}{t_{\text {react }}^{K}(p)}}{\frac{1}{t_{\text {react }}^{K}(o)}+\frac{1}{t_{\text {react }}^{K}(p)}}
$$

These estimates for the $\mathrm{p} / \mathrm{o}(T)$ are likely overestimates, as side reactions such as disproportionation ${ }^{41}$ are likely to occur.

When attempting to extract reaction rates from metadynamics simulations, it is good practice to observe whether the time distribution of the events, here the isomerization reaction, follows a Poisson distribution. ${ }^{37}$ The probability of observing a single event over an interval if the event follows a Poisson distribution of event rate $\tau$ is given by the exponential distribution $f_{T_{1}}(t)=\tau^{-1} \exp (t / \tau)$ with its associated cumulative distribution function $\operatorname{CDF}_{T_{1}}(t)=1-\exp (t / \tau)$. The empirical distribution function of the mean reaction times for a given system at a given temperature is fitted to $\operatorname{CDF}_{T_{1}}(t)$ to provide a robust estimate of the mean transition time $\tau$. The empirical cumulative functions and associated fits are shown in Figure S3. The quality of the fit is estimated using a Kolmogorov-Smirnov test, ${ }^{44}$ and the $p$-values associated with the fits shown in Figure $S 3$ are shown in Table 2. It

Table 2. $p$-Values for a Kolmogorov-Smirnov Test Performed on the Distributions Shown in Figure S3 and the Associated Fits with the Cumulative Distribution of a Poisson Distribution ${ }^{a}$

\begin{tabular}{cccccc} 
system & $300 \mathrm{~K}$ & $400 \mathrm{~K}$ & $500 \mathrm{~K}$ & $600 \mathrm{~K}$ & $700 \mathrm{~K}$ \\
\hline $\mathrm{MP}^{\ddagger}$-vac & 0.757 & 0.993 & 0.902 & 0.777 & 0.851 \\
$\mathrm{MP}^{\ddagger} @ \mathrm{CB} 6$ & 0.065 & 0.477 & 0.107 & 0.241 & 0.233 \\
$\mathrm{MP}^{\ddagger} @ \mathrm{CB} 7$ & 0.305 & 0.005 & 0.090 & 0.433 & 0.805 \\
$\mathrm{MO}^{\ddagger}$-vac & 0.262 & 0.709 & 0.728 & 0.962 & 0.946 \\
$\mathrm{MO}^{\ddagger} @ \mathrm{CB} 6$ & 0.649 & 0.195 & 0.328 & 0.334 & 0.471 \\
$\mathrm{MO}^{\ddagger} @ \mathrm{CB} 7$ & 0.038 & 0.085 & 0.340 & 0.155 & 0.234 \\
${ }^{a}$ Values for $t^{\mathrm{MTD}}$ & \multicolumn{5}{c}{ corresponding to $p$-values lower than 0.05 were }
\end{tabular}
discarded.

can be seen that $p$-values for simulations at low temperature are lower than those at high temperature. Additionally, simulations of $\mathrm{CB}$-enclosed reactions tend to yield lower $p$ values than the corresponding reactions in vacuum. A low $p$ value (below 0.05 ) has been reported to indicate an improper choice of collective variable or more generally a corruption of the dynamics of the system by the biasing potential. ${ }^{37}$ In the case of corruption of the dynamics of the system, $t^{\mathrm{MTD}}$ is no longer a reliable estimator of the real time to transition and tends to overestimate it significantly.

Vibrational Coupling in the $[m-X y l e n e+\mathrm{H}]^{+} @ C B 6$. Observing how an initial input of kinetic energy spreads to different modes within a molecule and how well it can be exchanged with the environment can provide information about the dynamic properties of a system. For example, the energy exchange through vibrational coupling between hosts and guests in noncovalent complexes has been reported to be both fast and slow. Ultrafast vibrational cooling of guests has been reported ${ }^{45}$ while reduced radiationless decay rates have been observed in CB7. ${ }^{46}$ Removal of vibrational energy from a 
molecule in a solvent is likely to involve a contribution from molecule-solvent vibrational coupling. ${ }^{47}$ Additionally, proteins have been shown to be able to concentrate vibrational energy on specific parts of their structure. ${ }^{48}$ Ultrafast reaction rates have been shown to deviate from transition state theory (TST) rates in part due to interaction (friction) with the solvent through a phenomenon known as the dynamic solvent effect. ${ }^{49}$ Reports of solvent viscosity limiting TST rates in the case of isomerization of organic molecules through its influence on cavity forming have also been published. ${ }^{50}$ More generally, the TST in solvents is expected to depart from its original formulation in vacuum, where $\kappa$ in eq 3 is assumed to be unity, due to the occurrence of reaction barrier recrossing originating from the friction with solvent molecules. ${ }^{22}$

While it is expected that the interactions between a guest molecule and molecules in the surrounding media will be significantly altered upon encapsulation, a qualitative picture of the new system would greatly help the understanding of intracavity reactions, a.k.a. inner-phase reactions. Given how much influence vibrational coupling between a molecule and its environment can have, it would be very interesting to study and measure how cucurbiturils, which are in direct contact with the solvent, can exchange energy with an encapsulated guest. Additionally, since solvent molecules and the guest become physically segregated upon guest encapsulation, their interactions and possible modulation of the barrier recrossing phenomenon needs to be mediated by the host, often through host-guest vibrational coupling. From this perspective, it is expected that the study of host-guest vibrational coupling can provide valuable insights.

As the $[m \text {-xylene }+\mathrm{H}]^{+} @$ CB6 system was revealed to be the most promising after the metadynamics study of the isomerization reaction, it was selected for further investigation. First, the 375 modes of the $[m \text {-xylene }+\mathrm{H}]^{+} @$ CB6 were assigned to either the $[m \text {-xylene }+\mathrm{H}]^{+}$guest $(28$ modes with $S$ $\geq 0.9)$, the CB6 host (258 modes with $S \leq 0.1$ ) or labeled as mixed ( 89 modes with $0.1 \leq S \geq 0.9$ ) using eq $S 5$. The mode assignment to either the guest, host, or mixed is shown in Figures S6-S9 for the systems m-xylene@CB6, [m-xylene + $\mathrm{H}]^{+} @ \mathbf{C B 6}, m$-xylene@CB7, and [m-xylene + H $]^{+} @ \mathbf{C B} 7 .^{51}$ Then, the relaxation rates $\gamma_{k}$ of each modes of the complex were computed by solving the system defined by ${ }^{52}$ eq 5 (refer to eq S4 in section 1.3 in the Supporting Information for details).

$$
\begin{aligned}
\gamma_{k} & =\frac{1}{16} \sum_{i=1, j=1}^{s} X_{i j k}^{2}\left\{\frac{\left(n_{i}+n_{j}+1\right)\left(\gamma_{i}+\gamma_{j}+\gamma_{k}\right)}{\left(\omega_{i}+\omega_{j}-\omega_{k}\right)^{2}+\left(\gamma_{i}+\gamma_{j}+\gamma_{k}\right)^{2} / 4}\right. \\
& +\frac{\left(n_{i}+n_{j}+1\right)\left(\gamma_{i}+\gamma_{j}+\gamma_{k}\right)}{\left(\omega_{i}+\omega_{j}+\omega_{k}\right)^{2}+\left(\gamma_{i}+\gamma_{j}+\gamma_{k}\right)^{2} / 4} \\
& +\frac{\left(n_{j}-n_{i}\right)\left(\gamma_{i}+\gamma_{j}+\gamma_{k}\right)}{\left(\omega_{i}-\omega_{j}-\omega_{k}\right)^{2}+\left(\gamma_{i}+\gamma_{j}+\gamma_{k}\right)^{2} / 4} \\
& +\frac{\left(n_{i}-n_{j}\right)\left(\gamma_{i}+\gamma_{j}+\gamma_{k}\right)}{\left(\omega_{j}-\omega_{i}-\omega_{k}\right)^{2}+\left(\gamma_{i}+\gamma_{j}+\gamma_{k}\right)^{2} / 4}
\end{aligned}
$$

Each $\gamma_{k}$ is subsequently broken down in six terms based on the terms of the sum inside of eq 5 . Indeed, each term of the sum quantifies the contribution of mode $i$ and $j$ to the relaxation rate of mode $k$ via the coupling coefficient $X_{i j k}$ and their respective frequencies. The Boltzmann's population of the $i$ th mode, with frequency $\omega_{i}$, is represented by $n_{i}$. As can be seen on eq 5 , each $\gamma_{k}$ implicitly depends on its own associated frequency $\omega_{k}$ in addition to two other frequencies $\omega_{j}$ and $\omega_{i}$. Since through eq S5, it is possible to assign $\omega_{j}$ and $\omega_{i}$ to either the guest, host, or a mix of both via their associated eigenvectors, it is possible to approximately keep track of where energy relaxing from mode $k$ is redistributed. A contribution to the relaxation rate of $k$ to modes $i$ and $j$, where both $\omega_{j}$ and $\omega_{i}$ are assigned to $[m \text {-xylene }+\mathrm{H}]^{+}$would be added to the $[m \text {-xylene }+\mathrm{H}]^{+}$specific relaxation rate of mode $k$ written as $\gamma_{k}^{x x}$. Similarly, a contribution from mode $i$, belonging to the CB6 assigned modes, and $j$, belonging to the mixed modes, would be written as $\gamma_{k}^{c m}$. The four last possible types of contribution are labeled as $\gamma_{k}^{c c}, \gamma_{k}^{m m}, \gamma_{k}^{x m}, \gamma_{k}^{x c}$, where $i$ belongs to CB6, mixed, $[m \text {-xylene }+\mathrm{H}]^{+}$, and $[m \text {-xylene }+\mathrm{H}]^{+}$ assigned modes, respectively, and $j$ belongs to $\mathbf{C B 6}$, mixed, and mixed and CB6 assigned modes, respectively. The breakdown of $\gamma_{k}$ into its components is illustrated in Figure S4 in the special case where the mode $k$ th has been assigned to the guest for clarity. The total relaxation rate involving $[m \text {-xylene }+\mathrm{H}]^{+}$ labeled modes is computed as $\gamma_{k}^{x x}+\gamma_{k}^{x c}+\gamma_{k}^{x m}$, while the total relaxation rate is simply $\gamma_{k}^{x x}+\gamma_{k}^{x c}+\gamma_{k}^{x m}+\gamma_{k}^{c c}+\gamma_{k}^{m m}+\gamma_{k}^{c m}$; both are shown in Figure 3 for systems $[m \text {-xylene }+\mathrm{H}]^{+} @$ CB6 and $[m \text {-xylene }+\mathrm{H}]^{+} @ \mathbf{C B} 7$ and on Figure S5 for the corresponding neutral systems m-xylene@CB6 and $m$ xylene@CB7.
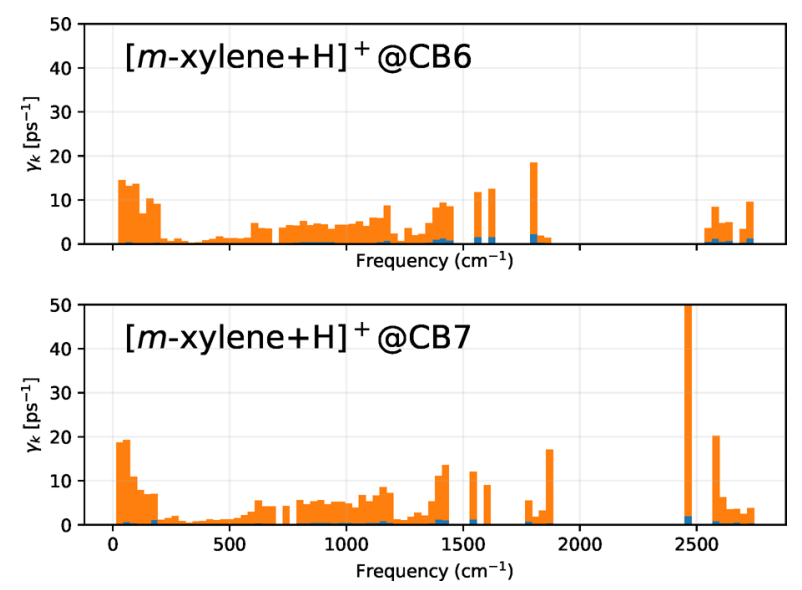

Figure 3. Anharmonic relaxation rates for the systems $[m$-xylene + $\mathrm{H}]^{+} @$ CB6 and $[m \text {-xylene }+\mathrm{H}]^{+} @$ CB7. The guest specific relaxation rate $\gamma_{k}^{x x}+\gamma_{k}^{x c}+\gamma_{k}^{x m}$ is shown in solid blue at the bottom while the overall relaxation rate is shown in orange on top. The decay rates are binned by $30 \mathrm{~cm}^{-1}$ with the bar height corresponding to the mean value of the bin.

From Figure 3, it appears that the guest specific relaxation rate is often significantly lower than the overall relaxation rates. Both relaxation rates are mostly of the order of $1-10 \mathrm{ps}^{-1}$, which is on the upper range though consistent with literature values. ${ }^{53,54}$ The relatively large relaxation rates are attributed to the large cubic coupling coefficients obtained from the numerical computation. From the study of the anharmonic relaxation rates, it appears that relaxation rates of the complex to the $[m \text {-xylene }+\mathrm{H}]^{+}$guest are expected to be relatively small, likely hindering energy transfer through that mechanism.

In order to test this hypothesis, the energy transfer from a mode majorly located on CB6 $(S=0.001)$ is observed. Mode 
(a)

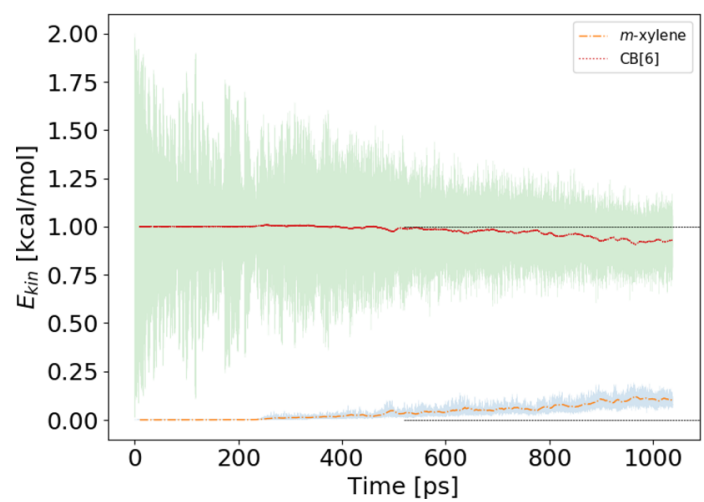

(b)

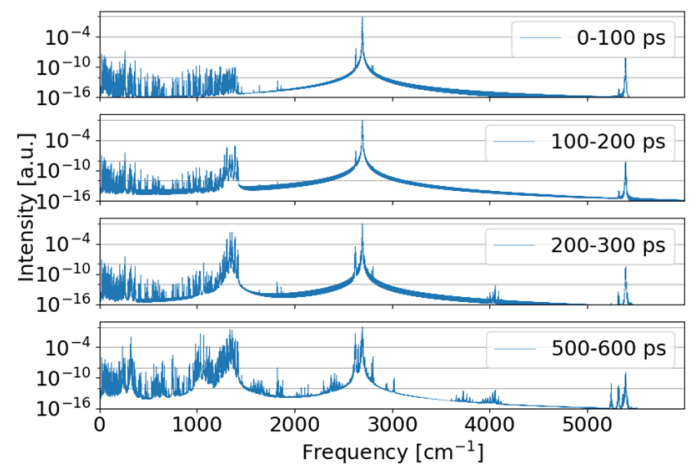

Figure 4. (a) Time evolution of the kinetic energy of the CB6 fragment and of the $[m \text {-xylene }+\mathrm{H}]^{+}$fragment over a 1 ns trajectory. After a significant lag period that involves intramolecular energy redistribution in CB6, energy is finally transmitted to the $[m \text {-xylene }+\mathrm{H}]^{+}$guest from 200 ps and onward. Moving average with a $10 \mathrm{ps}$ window is shown as dashed trendlines. (b) Frequency spectrum obtained from the autocorrelation of the velocity vector over various section of the $[m \text {-xylene }+\mathrm{H}]^{+} @ \mathbf{C B 6}$ system. It can be seen that soon after the deposition of $1 \mathrm{kcal} / \mathrm{mol}$ of energy on a mode predominantly present in CB6 at around $2700 \mathrm{~cm}^{-1}$ (mode 368), the energy shifts to a mode of approximately half the base frequency at around $1350 \mathrm{~cm}^{-1}$.

368 has a predicted relaxation rate to $[m \text {-xylene }+\mathrm{H}]^{+}$of 0.99 $\mathrm{ps}^{-1}$ and an overall relaxation rate of $4.77 \mathrm{ps}^{-1}$. After deposition of $1 \mathrm{kcal} / \mathrm{mol}$ on mode 368 mode of the MP $₫$ CB6 system located at $2671.37 \mathrm{~cm}^{-1}$, the rate of energy transfer is monitored via the quantity of kinetic energy associated with each subsystem, $[m \text {-xylene }+\mathrm{H}]^{+}$and CB6. The time evolution of the kinetic energy for both systems is shown in Figure 4a. It should be noted that the sum of kinetic energy of both subsystems does not sum to unity at the end of the simulation due to a small and likely unavoidable energy drift. If the vibrational energy was being ideally distributed between the host (108 atoms) and the guest (19 atoms) based on their atom numbers, one would expect around $15 \%$ of the kinetic energy to end up located on the guest. Toward the end of the trajectory, around $12 \%$ of the total kinetic energy is located on the guest which is not too far from the estimates.

Interestingly, it can be seen that the kinetic energy undergoes large fluctuations at the beginning of the trajectory. The kinetic energy fluctuations in the microcanonical ensemble should be constant at equilibrium and given by ${ }^{55}$

$$
\left\langle E_{\text {kin }}^{2}\right\rangle-\left\langle E_{\text {kin }}\right\rangle^{2}=\frac{3 k_{\mathrm{B}}{ }^{2} T^{2}}{2 N}\left(1-\frac{3 k_{\mathrm{B}}}{2 C_{v}}\right)
$$

where $N$ is the atom number, $k_{\mathrm{B}}$ the Boltzmann's constant, $C_{\mathrm{v}}$ the specific heat at constant volume, and $T$ the temperature. The large initial fluctuations therefore appear to be a transient effect. Most likely, in the initial phase of the trajectory when the majority of the energy is located on a single mode (mode 368) with few interactions with other modes, mode 368 essentially behaves like an isolated harmonic oscillator where complete conversion from kinetic to potential energy is allowed to occur. As anharmonic effects become more pronounced and energy starts to flow toward other modes on the host, the system departs from this "isolated oscillator" behavior and converges toward equilibrium and its equilibrium kinetic energy fluctuations value.

Quite tellingly, only a minimal amount of kinetic energy is transferred from the CB6 host to the guest before at least 200 ps of simulation. This represents a long time compared to $\approx 10$ ps, found in the case of the fast coupling of host-guest complexes. $^{45}$ The mode chosen for the initial energy deposition (368th) is predominantly located on CB6 and involves mostly eight hydrogens located on the carbon bridges that link a single glycoluril unit to the other in CB6. These hydrogens are facing out of the cavity and it can be seen intuitively how this mode would be unlikely to interact directly with the guest. In fact it is known that for energy transfer to occur through anharmonic coupling, there should be spacial overlap of the modes involved. ${ }^{56}$

The power spectrum of the trajectory shown in Figure 4 is displayed in Figure $4 \mathrm{~b}$ and computed using eqs S7 and S8. The initial mode is clearly visible on the spectrum decomposition in the first picoseconds of the trajectory $(0-10 \mathrm{ps})$ and is located at $2696 \mathrm{~cm}^{-1}$, not too far away from the original target frequency of $2671.37 \mathrm{~cm}^{-1}$. The discrepancy between the measured and targeted frequency resides in the fact that the static frequency computation is based on the harmonic approximation and therefore likely to miss anharmonic effects that will be captured by the spectrum computed from the molecular dynamics trajectory. The frequency located at 5392 $\mathrm{cm}^{-1}$ corresponds to twice the excitation frequency and is likely a result of anharmonicity.

As spectra are computed for section of the trajectory further ahead, a broadening of the peak associated with the excitation frequency occurs. Cucurbiturils possess a highly degenerate vibrational spectrum as a consequence of their high degree of symmetry. Mode 368 is in fact degenerated in the free cucurbituril and corresponds to the oscillation of all the hydrogens located on the carbons bridging the CB6's glycoluril units instead of just 8 located the carbon bridges of a glycoluril unit. Breakdown of the degeneracy yields several modes located near each other in frequency and position. It appears reasonable that energy could be exchanged between modes located close to each other both spatially (atoms involved in the modes' motion lying close by) and in frequency (modes' frequency being close $){ }^{48}$ therefore leading to a broadening of the excitation peak in the velocity power spectrum.

The initial absence of intermolecular energy transfer in the first 200 ps of the trajectory could be an Anderson-like localization of vibrational energy which is known to occur in large molecules if the number of states mixed with the excited 
(a)

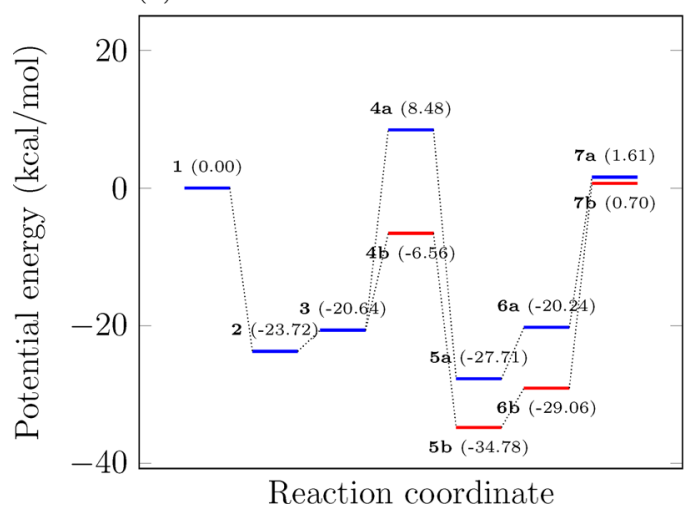

(b)

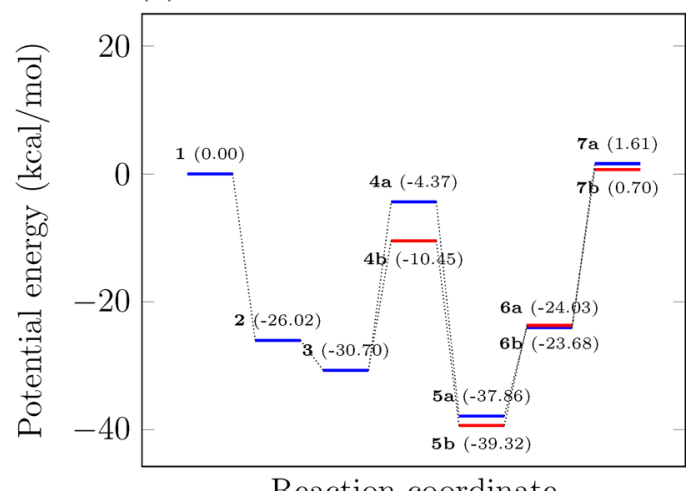

Figure 5. Overview of the energy landscape along a catalytic cycle in the case of (a) CB6 and (b) CB7. Potential energy evolution along the reaction path of $m$-xylene isomerization in CB toward $o$-xylene (pathway a on the scheme) and $p$-xylene (pathway $\mathbf{b}$ ). Values obtained using direct optimization at the PM6D3 level. The reference energy is the sum of the energies of $m$-xylene and $[\mathbf{C B}+\mathrm{H}]^{+}$separated and isolated in vacuum. Numbers corresponding to the step number are shown along with the corresponding energy in $\mathrm{kcal} / \mathrm{mol}$ between parentheses. The steps can be described as follows: first a neutral $m$-xylene molecule and $[\mathbf{C B} 6+\mathrm{H}]^{+}$are not interacting $(\mathbf{1})$ and then form a complex $(2)$. The proton is then shifted from the CB6 portal toward the $m$-xylene guest forming the complex $[m \text {-xylene }+\mathrm{H}]^{+} @ \mathbf{C B} 6$ at step (3). The isomerization reaction occurs with either transition state $\mathrm{MO}^{\ddagger} @ \mathbf{C B}$ (step 4a) or MP ${ }^{\ddagger} @ \mathbf{C B 6}$ (step 4b). At this point the system proceeds to either $[o \text {-xylene }+\mathrm{H}]^{+} @ \mathbf{C B}$ at step $(\mathbf{5 a})$ or $[p \text {-xylene }+\mathrm{H}]^{+} @ \mathbf{C B}$ at step $(\mathbf{5 b})$. Since the complex including protonated guests is too stable for the unbinding reaction to occur, the proton is shifted from the guest back to the cucurbituril yielding $o$-xylene@ $[\mathbf{C B}+\mathrm{H}]^{+}$at step $(\mathbf{6 a})$ and $p$-xylene@ $[\mathbf{C B}+\mathrm{H}]^{+}$at step $(\mathbf{6 b})$. At the last step, the system is able to dissociate, yielding the products $o$-xylene and $p$-xylene along with $[\mathbf{C B}+\mathrm{H}]^{+}$at steps $(7 \mathbf{a})$ and $(7 \mathbf{b})$, respectively.

state is low and the anharmonic coupling is weak. ${ }^{57,58}$ Transition from a regime where vibrational energy is localized on a specific mode to a regime where it is globally distributed, and the intermode energy transfer rate is comparatively high and has been labeled as the quantum ergodicity transition by some authors. ${ }^{58}$ It is tempting to attribute the sharp change in the rate of vibrational energy transfer seen in Figure $4 \mathrm{a}$ to a quantum ergodicity transition. The rate of decay and lifetime of a specific mode is system-dependent and depends on the availability of destination modes and of the third order anharmonic coupling coefficients between modes at first approximation, higher order harmonics being not negligible in general. ${ }^{53}$ Mode relaxation can be extremely slow and nanosecond scale is not unheard of. ${ }^{59,60}$ The rate of mode relaxation should not be influenced by the low effective temperature at such high frequencies, and the energy transfer is expected to go from high to intermediate frequencies rather than directly to low frequencies. ${ }^{56}$ This transfer to intermediate frequencies first is consistent with the results outlined in Figure $4 \mathrm{~b}$.

The power spectrum shown in Figure $4 \mathrm{~b}$ can be explained in terms of a cascade of relaxation rate starting with mode 368 . The main modes contributing to the relaxation rate of mode 368 are shown in Table S2. It can be seen that mode 368 primarily relaxes to other high frequency modes at neighboring frequencies (modes 331, 367, 375, 364, and 346) and very low frequencies (modes 1 and 2) along with an intermediate frequency (mode 318 ) located at $1815.97 \mathrm{~cm}^{-1}$. This decay to low frequencies and broadening of the mode 368 can be seen on the two top spectra of Figure $4 \mathrm{~b}$. Mode 318, shown in Table S3, on the other hand takes much longer to accumulate energy, becoming well visible only from 200 ps onward as visible on Figure $4 \mathrm{~b}$. Mode 318 itself in fact relaxes quickly to other high $(331,367)$ and low frequency $(1,2)$ modes while also relaxing quickly to mode 303 located at $1380.17 \mathrm{~cm}^{-1}$. In Figure $4 \mathrm{~b}$, it can be seen that mode 303, shown in Table S4, becomes well visible from 100 ps onward. Mode 303 also relaxes to high (modes 318, 331, 367) and low (modes 1 and
2) frequencies while also relaxing to neighboring frequencies (modes 301 and 304) contributing to its spectral broadening. Modes 1 (Table S5) and 2 (Table S6) both relax to relatively low frequencies with a high rate, mostly due to strong anharmonic coefficients, explaining the rapid appearance of spectral components below $500 \mathrm{~cm}^{-1}$.

As seen from Figure $4 \mathrm{a}$, the relatively slow energy transfer from mode 368 seems to indicate that vibrational energy located on the host is not easily transported to the guest. While thermal equilibration does occur on a time scale of $1 \mathrm{~ns}$, this time buffer could effectively shield the guest from its surroundings, providing a slow heating. The long time buffer in the case of the relatively tight $[m \text {-xylene }+\mathrm{H}]^{+} @ \mathbf{C 6}$ complex reveals that a tight host-guest fit does not necessarily impede the shielding abilities of the host.

It should be kept in mind however that mode 368 has a relatively high frequency, at $2696 \mathrm{~cm}^{-1}$, that is not easily thermally accessible and might not be representative of all modes. In particular, as seen in Figures S6-S9, low frequency modes are largely mixed and delocalized and could help transport vibrational energy in addition to the anharmonic coupling mechanism mainly considered here.

Residence Time of Xylene Isomers in CB6 and CB7. The nature of the dynamic equilibrium of the binding reaction is of great interest. An extremely stable complex could imply that the binding and release of a guest is a limiting factor in the overall rate of a cavity catalyzed reaction. On the other hand, a highly dynamic equilibrium would place the actual isomerization reaction as the rate limiting factor. An estimation of the magnitude of these rates enables the investigator to identify rate limiting bottlenecks that could benefit from further optimization.

During preliminary metadynamics simulation of xylene isomerization, it appeared that for temperatures above 700 $\mathrm{K}$, guests exiting the cavity tended to occur with increasing frequency even though no bias potential was applied to encourage a cavity exit. Indeed, a strong temperature dependence is expected for the binding affinity as the binding 
Table 3. Mean Exit Time at $300 \mathrm{~K}\left(t_{\text {exit }}^{300 \mathrm{~K}}\right), 400 \mathrm{~K}\left(t_{\text {exit }}^{400 \mathrm{~K}}\right), 500 \mathrm{~K}\left(t_{\text {exit }}^{500 \mathrm{~K}}\right), 600 \mathrm{~K}\left(t_{\text {exit }}^{600 \mathrm{~K}}\right)$ and Associated Enthalpy Barrier $\Delta H_{\text {exit }}^{\ddagger}$

\begin{tabular}{|c|c|c|c|c|c|}
\hline system & $t_{\text {exit }}^{300 \mathrm{~K}}(s)$ & $t_{\text {exit }}^{400 \mathrm{~K}}(\mathrm{~s})$ & $t_{\text {exit }}^{500 \mathrm{~K}}(\mathrm{~s})$ & $t_{\text {exit }}^{600 \mathrm{~K}}(\mathrm{~s})$ & $\Delta H_{\text {exit }}^{\ddagger}(\mathrm{kcal} / \mathrm{mol})$ \\
\hline$o$-xylene@ $[\text { CB6 + H }]^{+}$ & $1.44 \times 10^{-2}$ & $1.37 \times 10^{-6}$ & $8.59 \times 10^{-8}$ & $4.43 \times 10^{-9}$ & 16.82 \\
\hline$m$-xylene@ $[\text { CB6 + H }]^{+}$ & $1.73 \times 10^{-3}$ & $6.64 \times 10^{-7}$ & $1.14 \times 10^{-8}$ & $8.13 \times 10^{-10}$ & 16.62 \\
\hline$p$-xylene@ $[\mathrm{CB} 6+\mathrm{H}]^{+}$ & 2.72 & $3.41 \times 10^{-5}$ & $7.69 \times 10^{-7}$ & $2.48 \times 10^{-8}$ & 21.09 \\
\hline$o$-xylene@ $[\mathrm{CB} 7+\mathrm{H}]^{+}$ & $2.48 \times 10^{-5}$ & $2.19 \times 10^{-8}$ & $1.03 \times 10^{-8}$ & $9.93 \times 10^{-10}$ & 10.81 \\
\hline$m$-xylene@ $[\text { CB7 + H }]^{+}$ & $2.15 \times 10^{-5}$ & $5.07 \times 10^{-8}$ & $7.67 \times 10^{-9}$ & $8.69 \times 10^{-10}$ & 11.03 \\
\hline$p$-xylene@ $[\mathrm{CB} 7+\mathrm{H}]^{+}$ & $6.79 \times 10^{-6}$ & $2.73 \times 10^{-8}$ & $6.55 \times 10^{-9}$ & $6.07 \times 10^{-10}$ & 9.93 \\
\hline
\end{tabular}

reaction is associated with a major loss of rotational and translational entropy as seen in Table $S 7$ in the case of the $[m-$ xylene+H $]^{+} @$ CB6 complex, for example (values obtained using direct optimization of the geometries and obtained using the Rigid Rotor Harmonic Oscillator approximation on the obtained structures). It should be noted that from the values in Table S7, the main contributions from the entropy penalty are from the loss of translational and rotational entropies, in contrast to previous work. ${ }^{12}$ In fact, there is a gain in vibrational entropy, likely originating from an overall shift of the normal modes of the complex to lower frequencies upon binding compared to its individual components. ${ }^{61}$

As illustrated in Figure 5a,b, besides the actual isomerization reaction, the rate limiting step is the exiting of the products $o$ xylene and $p$-xylene from the cavities of $[\mathbf{C B} 6+\mathrm{H}]^{+}$or $[\mathbf{C B} 7$ $+\mathrm{H}]^{+}$. It is noted that direct exiting of the protonated forms $[o \text {-xylene }+\mathrm{H}]^{+}$and $[p \text {-xylene }+\mathrm{H}]^{+}$from the cavities of CB6 and CB7 is very unfavored as the protonated guest would need to overcome the strong Coulombic interaction of the positive charge with the cucurbituril's carbonyl groups at the portal region. In addition, CB6 and CB7 are better suited to accommodate a positive charge on their large portal whereas xylenes are required to disrupt their aromaticity at a significant energetic cost.

The steps $7 \mathbf{a}$ and $\mathbf{7 b}$ from Figure $5 \mathrm{a}, \mathbf{b}$ in addition to the dissociation reaction of $m$-xylene@ $[\mathrm{CB} 6+\mathrm{H}]^{+}$and $m$ xylene@ $[\mathbf{C B} 7+\mathrm{H}]^{+}$were investigated using metadynamics. From Table 3 (Eyring plot shown in Figure S10), it appears that the time of cavity exit for all xylene isomers exceeds the rates of isomerization (see Table S1) at least from $600 \mathrm{~K}$ and above. Above $600 \mathrm{~K}$, exit times are of the order of $1 \mathrm{~ns}$ and suggest that product and reagent diffusion to and from the cavity are not limiting factors in the overall isomerization pathway. Video 5 and Video 6 in the Supporting Information illustrate the cavity exiting process by, respectively, $o$-xylene and $p$-xylene from the CB6 cavity.

The values of $\Delta H_{\text {exit }}^{\ddagger}$ in Table 3 can be compared to the binding energies indicated in Figure 5a,b as steps (2), (6a), and $(6 \mathbf{b})$ obtained using direct minimization of the systems. The trend and magnitudes of the binding enthalpies are consistent in the case of the neutral guests exiting the CB6 complexes with $p$-xylene possessing a binding energy approximately $5 \mathrm{kcal} / \mathrm{mol}$ larger than that of $m$-xylene and $o$ xylene, the absolute values of the enthalpies being, however, about $4-7 \mathrm{kcal} / \mathrm{mol}$ lower than those estimated by direct energy minimization. This latter discrepancy probably originates from the fact that the guest is able to explore higher energy configurations during the MD trajectory where the metadynamic bias would be able to bring about a dissociation event at a lower energy than that of the minimum energy of the system. The correlation is not conserved in the case of CB7. The enthalpy barriers for the exit of the cavity in the case of CB7 are roughly half of what would be expected based on direct minimization, with $p$-xylene paradoxically having the lowest barrier to exit. Indeed while direct minimization conserves the overall regular pumpkin shape of CB7 with the guest inside, even in the case of low temperature molecular dynamics trajectories, CB7 loses its symmetry, often dramatically, and exhibits significant flexibility. The exploration of this additional area of the potential energy surface yields a cavity exiting pathway with a significantly different energy barrier. The flexibility displayed by CB7 during the trajectories suggests that this macrocycle might not be as rigid as previously thought. ${ }^{12}$ This situation is being seen in vacuum in the present case, and it would be very interesting to observe the effect of water on this symmetry loss and the implications for the predictability of host-guest binding affinities. The occurrence of CB7 flexibility and its impact on the energy barrier of the exiting reaction were not anticipated and highlight the relevance of (biased) molecular dynamics approaches in the case of complex potential landscapes.

\section{CONCLUSION}

The promising role of CB6 as a catalyst in the isomerization of $[m \text {-xylene }+\mathrm{H}]^{+}$to $[p \text {-xylene }+\mathrm{H}]^{+}$has been suggested using infrequent metadynamics. The macrocycle is expected to speed up the $\mathrm{MP}^{\ddagger}$ isomerization rate at $500 \mathrm{~K}$ by up to 2 orders of magnitude thanks to the stabilization of the transition state while inhibiting the competing $\mathrm{MO}^{\ddagger}$-yielding reaction pathway. In contrast, $\mathbf{C B} 7$ is not expected to be a very effective catalyst for these reactions. Analysis of the vibrational coupling in the case of the $[m \text {-xylene }+\mathrm{H}]^{+} @$ CB6 system revealed that the vibrational coupling of the high-frequency mode localized modes on CB6 were very ineffective in transferring energy to the guest likely due to a weak anharmonic coupling with modes located on the guest. This result offers a new perspective on the shielding effect of the cavity on encapsulated guests and hints at the possibility of using the macrocycle to protect guests and transition states from solvent effects. Eventually, it was revealed that at temperatures higher than $500 \mathrm{~K}$, the average residence time of the guests inside the cavity could be as low as $1 \mathrm{~ns}$ and always lower than the mean time to reaction. This exciting result indicates that, in contrast to commonly used zeolites that suffer from important reagent diffusion limitations, the macrocycles CB6 and CB7 are likely to experience very fast guest turnover, allowing for potentially high catalytic activities.

\section{ASSOCIATED CONTENT}

\section{(s) Supporting Information}

The Supporting Information is available free of charge at https://pubs.acs.org/doi/10.1021/acs.jpcc.0c02012.

Illustration of isomerization results using direct transition state optimization and additional details on the procedure in the case of $\mathrm{MO}^{\ddagger} @ \mathbf{C B 7}$; computational details on the isomerization of $m$-xylene in CB6 and 
CB7 via metadynamics including definition of the collective variables and obtained cumulative distributions of the times to reaction; computation of cubic coupling coefficients and details on the resolution of the system of coupled equation to obtain the vibrational decay rates; top destination modes and associated decay rates for a selection of modes; illustration of the breakdown of the decay rates based on the modes involved; anharmonic decay rates of $m$-xylene@CB6 and $m$-xylene@CB7; and details on the computation of complex unbinding rates using metadymanics and associated Eyring plots (PDF)

Video of the isomerization of $\mathrm{MO}^{\ddagger}$ in vacuum (MP4)

Video of the isomerization of $\mathrm{MO}^{\ddagger}$ in CB6 (MP4)

Video of the isomerization of $\mathrm{MP}^{\ddagger}$ in vacuum (MP4)

Video of the isomerization of $\mathrm{MP}^{\ddagger}$ in CB6 (MP4)

Video of $o$-xylene exiting $[\mathbf{C B} 6+\mathrm{H}]^{+}(\mathrm{MP} 4)$

Video of $p$-xylene exiting $[\mathbf{C B} 6+\mathrm{H}]^{+}(\mathrm{MP} 4)$

\section{AUTHOR INFORMATION}

\section{Corresponding Authors}

Yong-Wei Zhang - Institute of High Performance Computing, A*STAR, Singapore 138632; 이이이.org/0000-0001-72551678; Email: zhangyw@ihpc.a-star.edu.sg

Tung-Chun Lee - Institute for Materials Discovery and Department of Chemistry, Christopher Ingold Building, University College London (UCL), London WC1E 6BT, U.K. 이이.org/0000-0002-3163-0000; Email: tungchun.lee@ ucl.ac.uk

\section{Author}

Hugues Lambert - Institute of High Performance Computing, $A^{*}$ STAR, Singapore 138632; Department of Chemistry, Christopher Ingold Building and Institute for Materials Discovery, University College London (UCL), London WC1H OAJ, U.K.; $\odot$ orcid.org/0000-0002-2913-3937

Complete contact information is available at: https://pubs.acs.org/10.1021/acs.jpcc.0c02012

\section{Notes}

The authors declare no competing financial interest.

\section{ACKNOWLEDGMENTS}

H.L. and T.-C.L. are grateful to the studentship funded by the A*STAR-UCL Research Attachment Programme through the EPSRC Centre for Doctoral Training in Molecular Modelling and Materials Science (Grant EP/L015862/1). T.-C.L. are grateful to the Research Project Grant (Grant RPG-2016-393) funded by the Leverhulme Trust. The authors are grateful for fruitful discussions with David Leitner and Hari Pandey on the topic of anharmonic decay of vibrational modes. The authors acknowledge the use of the UCL Legion and Myriad High Performance Computing Facility (Legion@UCL, Myriad@ UCL), and associated support services, in the completion of this work. The authors are grateful to the UK Materials and Molecular Modelling Hub for computational resources, which is partially funded by EPSRC (Grant EP/P020194/1). This work was supported by the A*STAR Computational Resource Centre through the use of its high performance computing facilities. This work used the ARCHER UK National Supercomputing Service (http://www.archer.ac.uk).

\section{REFERENCES}

(1) Solomonsz, W. A.; Rance, G. A.; Harris, B. J.; Khlobystov, A. N. Competitive hydrosilylation in carbon nanoreactors: probing the effect of nanoscale confinement on selectivity. Nanoscale 2013, 5, $12200-12205$.

(2) Zheng, S.; Jentys, A.; Lercher, J. A. Xylene isomerization with surface-modified HZSM-5 zeolite catalysts: An in situ IR study. J. Catal. 2006, 241, 304-311.

(3) Zhao, H.; Sen, S.; Udayabhaskararao, T.; Sawczyk, M.; Kučanda, K.; Manna, D.; Kundu, P. K.; Lee, J.-W.; Král, P.; Klajn, R. Reversible trapping and reaction acceleration within dynamically self-assembling nanoflasks. Nat. Nanotechnol. 2016, 11, 82-88.

(4) Halls, M. D.; Schlegel, H. B. Chemistry inside carbon nanotubes: the Menshutkin SN2 reaction. J. Phys. Chem. B 2002, 106, 19211925.

(5) Lienhard, G. E. Enzymatic catalysis and transition-state theory. Science 1973, 180, 149-154.

(6) Yao, Y.; Fu, Q.; Zhang, Y. Y.; Weng, X.; Li, H.; Chen, M.; Jin, L.; Dong, A.; Mu, R.; Jiang, P.; Liu, L.; Bluhm, H.; Liu, Z.; Zhang, S. B.; Bao, X. Graphene cover-promoted metal-catalyzed reactions. Proc. Natl. Acad. Sci. U. S. A. 2014, 111, 17023-17028.

(7) Miners, S. A.; Rance, G. A.; Khlobystov, A. N. Chemical reactions confined within carbon nanotubes. Chem. Soc. Rev. 2016, 45, $4727-4746$.

(8) Renggli, K.; Nussbaumer, M. G.; Urbani, R.; Pfohl, T.; Bruns, N. A Chaperonin as protein nanoreactor for atom-transfer radical polymerization. Angew. Chem., Int. Ed. 2014, 53, 1443-1447.

(9) Yoshizawa, M.; Takeyama, Y.; Okano, T.; Fujita, M. Cavitydirected synthesis within a self-assembled coordination cage: Highly selective $[2+2]$ cross-photodimerization of olefins. J. Am. Chem. Soc. 2003, 125, 3243-3247.

(10) Arumugam, S.; Vutukuri, D. R.; Thayumanavan, S.; Ramamurthy, V. Amphiphilic homopolymer as a reaction medium in water: product selectivity within polymeric nanopockets. J. Am. Chem. Soc. 2005, 127, 13200-13206.

(11) Lee, T.-C.; Kalenius, E.; Lazar, A. I.; Assaf, K. I.; Kuhnert, N.; Grün, C. H.; Jänis, J.; Scherman, O. A.; Nau, W. M. Chemistry inside molecular containers in the gas phase. Nat. Chem. 2013, 5, 376-382.

(12) Rekharsky, M. V.; Mori, T.; Yang, C.; Ko, Y. H.; Selvapalam, N.; Kim, H.; Sobransingh, D.; Kaifer, A. E.; Liu, S.; Isaacs, L.; Chen, W.; Moghaddam, S.; Gilson, M. K.; Kim, K.; Inoue, Y. A synthetic host-guest system achieves avidin-biotin affinity by overcoming enthalpy-entropy compensation. Proc. Natl. Acad. Sci. U. S. A. 2007, 104, 20737-20742.

(13) Lambert, H.; Mohan, N.; Lee, T.-C. Ultrahigh binding affinity of a hydrocarbon guest inside cucurbit[7] uril enhanced by strong host-guest charge matching. Phys. Chem. Chem. Phys. 2019, 21, $14521-14529$.

(14) Mock, W.; Irra, T.; Wepsiec, J.; Manimaran, T. Cycloaddition induced by cucurbituril. A case of Pauling principle catalysis. J. Org. Chem. 1983, 48, 3619-3620.

(15) Wagner, A.; Ly, K. H.; Heidary, N.; Szabó, I.; Földes, T.; Assaf, K. I.; Barrow, S. J.; Sokołowski, K.; Al-Hada, M.; Kornienko, N.; et al. Host-guest chemistry meets electrocatalysis: cucurbit[6]uril on a $\mathrm{Au}$ surface as a hybrid system in CO2 reduction. ACS Catal. 2020, 10, $751-761$.

(16) Miskolczy, Z.; Megyesi, M.; Tárkányi, G.; Mizsei, R.; Biczók, L. Inclusion complex formation of sanguinarine alkaloid with cucurbit[7]uril: inhibition of nucleophilic attack and photooxidation. Org. Biomol. Chem. 2011, 9, 1061-1070.

(17) Ren, H.; Huang, Z.; Yang, H.; Xu, H.; Zhang, X. Controlling the reactivity of the Se-Se Bond by the supramolecular chemistry of cucurbituril. ChemPhysChem 2015, 16, 523-527.

(18) Jon, S. Y.; Ko, Y. H.; Park, S. H.; Kim, H.-J.; Kim, K. A facile, stereoselective $[2+2]$ photoreaction mediated by cucurbit[8]uril. Chem. Commun. 2001, 1938-1939.

(19) Yang, H.; Liu, Y.; Liu, K.; Yang, L.; Wang, Z.; Zhang, X. Rational adjustment of multicolor emissions by cucurbiturils-based 
host-guest chemistry and photochemistry. Langmuir 2013, 29, 12909-12914.

(20) Assaf, K. I.; Nau, W. M. Cucurbiturils: from synthesis to highaffinity binding and catalysis. Chem. Soc. Rev. 2015, 44, 394-418.

(21) Rozanska, X.; van Santen, R. A.; Hutschka, F.; Hafner, J. A periodic DFT study of intramolecular isomerization reactions of toluene and xylenes catalyzed by acidic mordenite. J. Am. Chem. Soc. 2001, 123, 7655-7667.

(22) Truhlar, D. G.; Garrett, B. C.; Klippenstein, S. J. Current status of transition-state theory. J. Phys. Chem. 1996, 100, 12771-12800.

(23) Passerone, D.; Ceccarelli, M.; Parrinello, M. A concerted variational strategy for investigating rare events. J. Chem. Phys. 2003, 118, 2025-2032.

(24) Iannuzzi, M.; Laio, A.; Parrinello, M. Efficient exploration of reactive potential energy surfaces using Car-Parrinello molecular dynamics. Phys. Rev. Lett. 2003, 90, 238302.

(25) Barducci, A.; Bonomi, M.; Parrinello, M. Metadynamics. Wiley Interdiscip. Rev.: Comput. Mol. Sci. 2011, 1, 826-843.

(26) Torrie, G. M.; Valleau, J. P. Nonphysical sampling distributions in Monte Carlo free-energy estimation: Umbrella sampling. J. Comput. Phys. 1977, 23, 187-199.

(27) Müller, E. M.; de Meijere, A.; Grubmüller, H. Predicting unimolecular chemical reactions: Chemical flooding. J. Chem. Phys. 2002, 116, 897-905.

(28) Darve, E.; Pohorille, A. Calculating free energies using average force. J. Chem. Phys. 2001, 115, 9169-9183.

(29) Polino, D.; Parrinello, M. Combustion chemistry via metadynamics: Benzyl decomposition revisited. J. Phys. Chem. A 2015, 119, 978-989.

(30) Limongelli, V.; Bonomi, M.; Parrinello, M. Funnel metadynamics as accurate binding free-energy method. Proc. Natl. Acad. Sci. U. S. A. 2013, 110, 6358-6363.

(31) Tiwary, P.; Limongelli, V.; Salvalaglio, M.; Parrinello, M. Kinetics of protein-ligand unbinding: Predicting pathways, rates, and rate-limiting steps. Proc. Natl. Acad. Sci. U. S. A. 2015, 112, E386E391.

(32) Fleming, K. L.; Tiwary, P.; Pfaendtner, J. New approach for investigating reaction dynamics and rates with ab initio calculations. $J$. Phys. Chem. A 2016, 120, 299-305.

(33) Fu, C. D.; Oliveira, L. F. L.; Pfaendtner, J. Determining energy barriers and selectivities of a multi-pathway system with infrequent metadynamics. J. Chem. Phys. 2017, 146, 014108.

(34) Tiwary, P.; Parrinello, M. From metadynamics to dynamics. Phys. Rev. Lett. 2013, 111, 230602.

(35) Laidler, K. J.; King, M. C. Development of transition-state theory. J. Phys. Chem. 1983, 87, 2657-2664.

(36) McQuarrie, D. A. Statistical Mechanics; Harper \& Row Publishers, 1976.

(37) Salvalaglio, M.; Tiwary, P.; Parrinello, M. Assessing the reliability of the dynamics reconstructed from metadynamics. J. Chem. Theory Comput. 2014, 10, 1420-1425.

(38) Nau, W. M.; Florea, M.; Assaf, K. I. Deep inside cucurbiturils: physical properties and volumes of their inner cavity determine the hydrophobic driving force for host-guest complexation. Isr. J. Chem. 2011, 51, 559-577.

(39) Ajami, D.; Tolstoy, P. M.; Dube, H.; Odermatt, S.; Koeppe, B.; Guo, J.; Limbach, H.-H.; Rebek, J., Jr Encapsulated carboxylic acid dimers with compressed hydrogen bonds. Angew. Chem., Int. Ed. 2011, 50, 528-531.

(40) Brown, H. C.; Jungk, H. The isomerization of o-and p-Xylenes and some related alkylbenzenes under the influence of hydrogen bromide and aluminum bromide; the relative isomerization aptitudes of alkyl Groups. J. Am. Chem. Soc. 1955, 77, 5579-5584.

(41) Jones, C. W.; Zones, S. I.; Davis, M. E. m-Xylene reactions over zeolites with unidimensional pore systems. Appl. Catal., A 1999, 181, 289-303.

(42) Bauer, F.; Chen, W.-H.; Ernst, H.; Huang, S.-J.; Freyer, A.; Liu, S.-B. Selectivity improvement in xylene isomerization. Microporous Mesoporous Mater. 2004, 72, 81-89.
(43) Kärger, J.; Vasenkov, S.; Auerbach, S. M. Handbook of Zeolite Science and Technology; CRC Press, 2003; pp 458-560.

(44) Massey, F. J., Jr The Kolmogorov-Smirnov test for goodness of fit. J. Am. Stat. Assoc. 1951, 46, 68-78.

(45) Aulin, Y. V.; Liu, M.; Piotrowiak, P. Ultrafast vibrational cooling inside of a molecular container. J. Phys. Chem. Lett. 2019, 10, 24342438.

(46) Mohanty, J.; Nau, W. M. Ultrastable rhodamine with cucurbituril. Angew. Chem., Int. Ed. 2005, 44, 3750-3754.

(47) Magde, D.; Rojas, G. E.; Seybold, P. G. Solvent dependence of the fluorescence lifetimes of xanthene dyes. Photochem. Photobiol. 1999, 70, 737-744.

(48) Morgan, S. E.; Cole, D. J.; Chin, A. W. Nonlinear network model analysis of vibrational energy transfer and localisation in the Fenna-Matthews-Olson complex. Sci. Rep. 2016, 6, 36703.

(49) Barbara, P. F.; Jarzeba, W. Dynamic solvent effects on polar and nonpolar isomerizations. Acc. Chem. Res. 1988, 21, 195-199.

(50) Sumi, H. Solvent fluctuations and viscosity-dependent rates of solution reactions in a regime indescribable by the transition state theory. J. Mol. Liq. 2001, 90, 185-194.

(51) Leitner, D. M.; Pandey, H. D. Asymmetric energy flow in liquid alkylbenzenes: A computational study. J. Chem. Phys. 2015, 143, 144301 .

(52) Stuchebrukhov, A.; Kuzmin, M.; Bagratashvili, V.; Letokhov, V. Threshold energy dependence of intramolecular vibrational relaxation in polyatomic molecules. Chem. Phys. 1986, 107, 429-443.

(53) Yu, X.; Leitner, D. M. Vibrational energy transfer and heat conduction in a protein. J. Phys. Chem. B 2003, 107, 1698-1707.

(54) Pandey, H. D.; Leitner, D. M. Vibrational states and nitrile lifetimes of cyanophenylalanine isotopomers in solution. J. Phys. Chem. A 2018, 122, 6856-6863.

(55) Smith, B.; Frenkel, D. Understanding Molecular Simulations; Academic: New York, 1996.

(56) Leitner, D. M. Energy flow in proteins. Annu. Rev. Phys. Chem. 2008, 59, 233-259.

(57) Stuchebrukhov, A.; Marcus, R. Theoretical study of intramolecular vibrational relaxation of acetylenic $\mathrm{CH}$ vibration for $\mathrm{v}=1$ and 2 in large polyatomic molecules $(\mathrm{CX} 3) 3 \mathrm{YCCH}$, where $\mathrm{X}=\mathrm{H}$ or $\mathrm{D}$ and $\mathrm{Y}=\mathrm{C}$ or Si. J. Chem. Phys. 1993, 98, 6044-6061.

(58) Leitner, D. M.; Wolynes, P. G. Vibrational relaxation and energy localization in polyatomics: Effects of high-order resonances on flow rates and the quantum ergodicity transition. J. Chem. Phys. 1996, 105, 11226-11236.

(59) Xie, A.; Van Der Meer, A. F.; Austin, R. H. Excited-state lifetimes of far-infrared collective modes in proteins. Phys. Rev. Lett. 2001, 88, 018102.

(60) Bigwood, R.; Gruebele, M. A simple matrix model of intramolecular vibrational redistribution and its implications. Chem. Phys. Lett. 1995, 235, 604-613.

(61) Tidor, B.; Karplus, M. The contribution of vibrational entropy to molecular association: the dimerization of insulin. J. Mol. Biol. 1994, 238, 405-414. 